\title{
ESTIMATIVA DA PRODUÇÃO ANUAL DE SERAPILHEIRA DOS BOSQUES DE MANGUE NO FURO GRANDE, BRAGANÇA-PARÁ ${ }^{1}$
}

Marcus Emanuel Barroncas Fernandes ${ }^{2}$, Antonia Aparecida Monteiro do Nascimento ${ }^{3}$ e Muzenilha Lira

Carvalho ${ }^{4}$

\begin{abstract}
RESUMO - As condições ambientais de determinado local podem influenciar a produtividade dos manguezais. Assim, este estudo estimou a produção total e dos componentes da serapilheira no Furo Grande, Bragança, PA. Este estudo compreendeu quatro ciclos anuais (julho/2000 a agosto/2004) em três sítios. Foram instaladas sete cestas em cada sítio ao longo de uma transecção de $140 \mathrm{~m}$, com intervalos de $20 \mathrm{~m}$. Cada cesta possuía uma área útil de $1 \mathrm{~m}^{2}$, com tela de $1 \mathrm{~mm}^{2}$, suspensa acima do nível das marés de sizígia. O material acumulado nas cestas foi coletado mensalmente, separado em folha, flor, fruto, estípula, galho e miscelânea, sendo posteriormente secado a $70^{\circ} \mathrm{C}$ até alcançar peso constante. A produção média dos quatro anos foi de 9,85 t.ha $^{-1}$.ano ${ }^{-1}$ no sítio 1, 6,41 tha- ${ }^{-1}$.ano ${ }^{-1}$ no sítio 2 e 5,99 t.ha- ${ }^{-1}$.ano $o^{-1}$ no sítio 3, cuja comparação apresenta diferença significativa entre os sítios 1 e $3(\mathrm{H}=7,53 ; \mathrm{gl}=2 ; \mathrm{p}<0,05)$. Em suma, os resultados apontaram que a folha foi o componente de maior produtividade e, juntamente com a flor, teve pico na estação seca, o que parece favorecer uma economia de energia para o investimento em reprodução, enquanto a maior produção de fruto foi na estação chuvosa, promovendo a dispersão de propágulos e, conseqüentemente, a renovação e manutenção dessas florestas.
\end{abstract}

Palavras-chave: Produção de serapilheira, manguezal e Furo Grande.

\section{ESTIMATES OF THE ANNUAL LITTER PRODUCTION IN MANGROVE STANDS IN FURO GRANDE, BRAGANÇA-PARÁ}

\begin{abstract}
It is well known that environmental conditions of a determined place can influence the productivity of mangroves. So, the present study estimated the total and components litter production in Furo Grande, Bragança-PA. This study comprised four annual cycles (July/2000 to August/2004) at three sites. Seven traps were placed at each site along a $140 \mathrm{~m}$ transect, with $20 \mathrm{~m}$ intervals. Each trap had a useful area of 1 $\mathrm{m}^{2}$, with $1 \mathrm{~mm}^{2}$ mesh, suspended above the spring tide level. Accumulated material in the traps was collected on a monthly basis, sorted manually into leaves, flowers, fruits, stipules, twigs, and miscellaneous and then

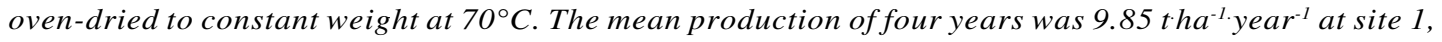

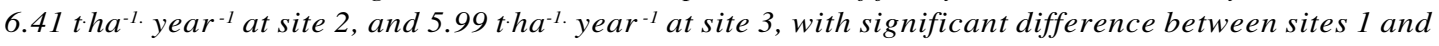
$3(H=7.53 ; d f=2 ; p<0.05)$. Overall, the results showed that leaf was the most productive component, and together with flower, had peak in the dry season, which seems to favor energy saving to invest in reproduction, whereas fruit peak in the wet season, providing propagule dispersion and hence the renewal and maintenance of these forests.
\end{abstract}

Keywords: Litter production, mangrove and Furo Grande.

\footnotetext{
${ }^{1}$ Recebido em 27.09.2006 e aceito para publicação em 05.06.2007.

${ }^{2}$ Universidade Federal do Pará, Campus de Bragança. Bragança-PA. E-mail: <mebf@ufpa.br>.

${ }^{3}$ Programa de Pós-Graduação em Biologia Ambiental da Universidade Federal do Pará. E-mail: <aamncida2001 @ yahoo.com.br>.

${ }^{4}$ Governo do Estado de Tocantins, Secretaria de Estado da Saúde, Palmas-TO . E-mail: <muzenilha@yahoo.com.br>.
} 


\section{INTRODUÇÃO}

As espécies arbóreas do manguezal desempenham papel fundamental na ciclagem de nutrientes, atuando como fonte primária de matéria orgânica, através da queda da serapilheira (AMARASINGHE e BALASUBRAMANIAN, 1992). A serapilheira é formada pelo acúmulo de material animal e, principalmente, por material vegetal decíduo, armazenado no sedimento da floresta (SILVA, 1984). Essa matéria orgânica acumulada no solo da floresta decompõe-se, suprindo o solo e as raízes com nutrientes (ARATO et al., 2003). De maneira geral, as florestas de mangue apresentam alta produção de serapilheira na região equatorial (SAENGER e SNEDAKER, 1993). Esse fato provavelmente decorra dos fatores relativos à latitude, como as altas taxas de insolação e pluviosidade e, principalmente, à disponibilidade de água doce (POOL et al., 1975).

De acordo com Day Jr. et al. (1987), parece haver uma tendência geral para o pico de produção da serapilheira nos manguezais ocorrer no período chuvoso, facilitando, assim, a exportação desse material para as áreas adjacentes. No entanto, picos de produção de serapilheira na estação seca foram registrados em estudos realizados nos manguezais da costa amazônica, naAmérica do Sul (CHALE, 1996; FERNANDES, 2003; MEHLIG 2006). Aalta produção no período seco, segundo Amarasinghe e Balasubramanian (1992), pode ser atribuída ao maior custo energético requerido para a planta manter os tecidos fotossintetizantes em condições ambientais de alta salinidade durante essa estação, causando, assim, a abscisão do maior número de folhas, que é o principal componente da serapilheira (FERNANDES, 2003).

As condições ambientais locais assumem importante papel na determinação dos padrões de produção de serapilheira no ecossistema de manguezal (FERNANDES, 2003). No entanto, os trabalhos realizados nos manguezais da Amazônia brasileira não apresentam séries de dados que possam descrever a existência de determinado padrão anual dessa produção. Assim, este estudo, através do registro ininterrupto de quatro anos de trabalho de campo, teve como objetivo verificar a existência de um padrão para a produção de serapilheira em uma floresta de mangue no litoral amazônico, ou seja, determinar quais os períodos de maior produção total e dos componentes da serapilheira e se esses períodos apresentam um padrão anual de produção, dada a ausência de informações acerca do monitoramento dessa produção na Amazônia brasileira.

\section{MATERIAL E MÉTODOS}

2.1. Descrição da área de estudo - Este estudo foi realizado no Furo Grande, entre as coordenadas $00^{\circ} 50^{\prime} 19,5^{\prime \prime} \mathrm{S}$ e 46³8' 14,9" W, localizado na península de Ajuruteua entre os rios Caeté e Taperaçu, no Município de Bragança-Pará (Figura 1). A área de estudo é inundada somente durante as marés de sizígia. O regime de maré é semidiurno, em que a altura máxima corresponde a $5 \mathrm{~m}$ durante as marés de sizígia (KOCH, 1999). A pluviosidade média anual na região da península alcança 3.000 $\mathrm{mm}$, com a umidade relativa do ar variando entre 80 e $91 \%$ (MARTORANO et al., 1993). O período seco inclui os meses de julho a dezembro, enquanto o período chuvoso ocorre nos meses de janeiro a junho.

2.2. Caracterização dos sítios de trabalho - A área de estudo foi subdividida em três sítios de trabalho, com base na sua formação vegetal. O sítio 1 é um bosque misto constituído, principalmente, por indivíduos de A. germinans (52,3\%) e $R$. mangle (47,7\%). O sítio 2 é um bosque com dominância de indivíduos de $A$. germinans $(93,1 \%)$, ao passo que no sítio $3 R$. mangle é a espécie dominante $(90,3 \%)$ (SEIXAS et al., 2006).

2.3. Produção da serapilheira - Parcelas de $10 \times 140 \mathrm{~m}$ foram abertas em cada sítio de trabalho para sorteio e delimitação das transecções. Uma transecção de $140 \mathrm{~m}$ foi, então, sorteada em cada sítio, paralela ao curso do furo (canal) e $50 \mathrm{~m}$ distante da rodovia e do furo para eliminar o efeito de borda.

Em cada sítio foram fixadas sete cestas coletoras $(n=21)$ em intervalos regulares de $20 \mathrm{~m}$, no período de agosto de 2000 a julho de 2004. Cada cesta possuía uma área útil de $1 \mathrm{~m}^{2}$, confeccionada com tela de náilon (malha de $1 \mathrm{~mm}$ ) e armação de madeira na borda (15 cm de altura), permanecendo suspensa acima do nível das marés de sizígia por estacas de madeira. O material acumulado nas cestas foi coletado mensalmente e armazenado em sacos plásticos para posterior triagem. No laboratório, o material foi triado manualmente nos seguintes componentes: folha, flor, fruto, galho, estípula e miscelânea. Desses, três componentes (folha, flor e fruto) foram identificados e separados por espécie (A. germinans e $R$. mangle). O componente galho não foi separado por espécie, pois não apresentou características que permitissem a identificação das espécies de mangue. O componente miscelânea 
representou todo material vegetal e animal não identificado, inclusive fezes. Esse procedimento foi executado para cada cesta coletora em separado, sendo o material de cada componente secado em estufa a $70^{\circ} \mathrm{C}$ até atingir peso constante, quando foi tomado o peso de matéria seca final por componente.

2.4. Análise de dados - As variações das taxas anuais de produção de serapilheira (total e componentes) foram comparadas através do teste nãoparamétrico de Kruskal-Wallis. Para as análises da variação da produção das folhas por espécie foi usado o teste ANOVA (fatorial a x b - com replicações). $\mathrm{O}$ teste não-paramétrico de Friedman foi utilizado para calcular a variação sazonal de cada sítio e de cada componente ao longo dos quatro anos. Para analisar as relações entre a produção de serapilheira de cada sítio e a variável precipitação foi empregada a análise de regressão linear. Todas as análises foram realizadas no pacote estatístico BioEstat 3.0 (AYRES et al., 2003).

\section{RESULTADOS}

A produção anual de serapilheira alcançou um valor médio de 7,4 t.ha- ${ }^{-1}$. ano ${ }^{-1}$, ressaltando-se que na comparação da produção total entre os sítios, para cada ano, apenas o ano 2 não apresentou diferença significativa (Kruskal-Wallis, $\mathrm{H}=5,36$; $\mathrm{gl}=2$; p $>0,05$ ) (Tabela 1). Da mesma forma, nenhum dos componentes apresentou variação na sua produção ao longo dos quatro anos estudados. Entretanto, a comparação da produção dos componentes entre si mostrou diferença bastante significativa (KruskalWallis, $\mathrm{p}<0,001)$.

O sítio 1 (R. mangle + A. germinans) foi o que apresentou a mais alta taxa de produção de serapilheira, seguido pelo sítio 2 (A. germinans) e sítio 3 ( $R$. mangle), exceto no ano 4 , quando o sítio 3 foi mais produtivo que o sítio 2 (Tabela 1 ).

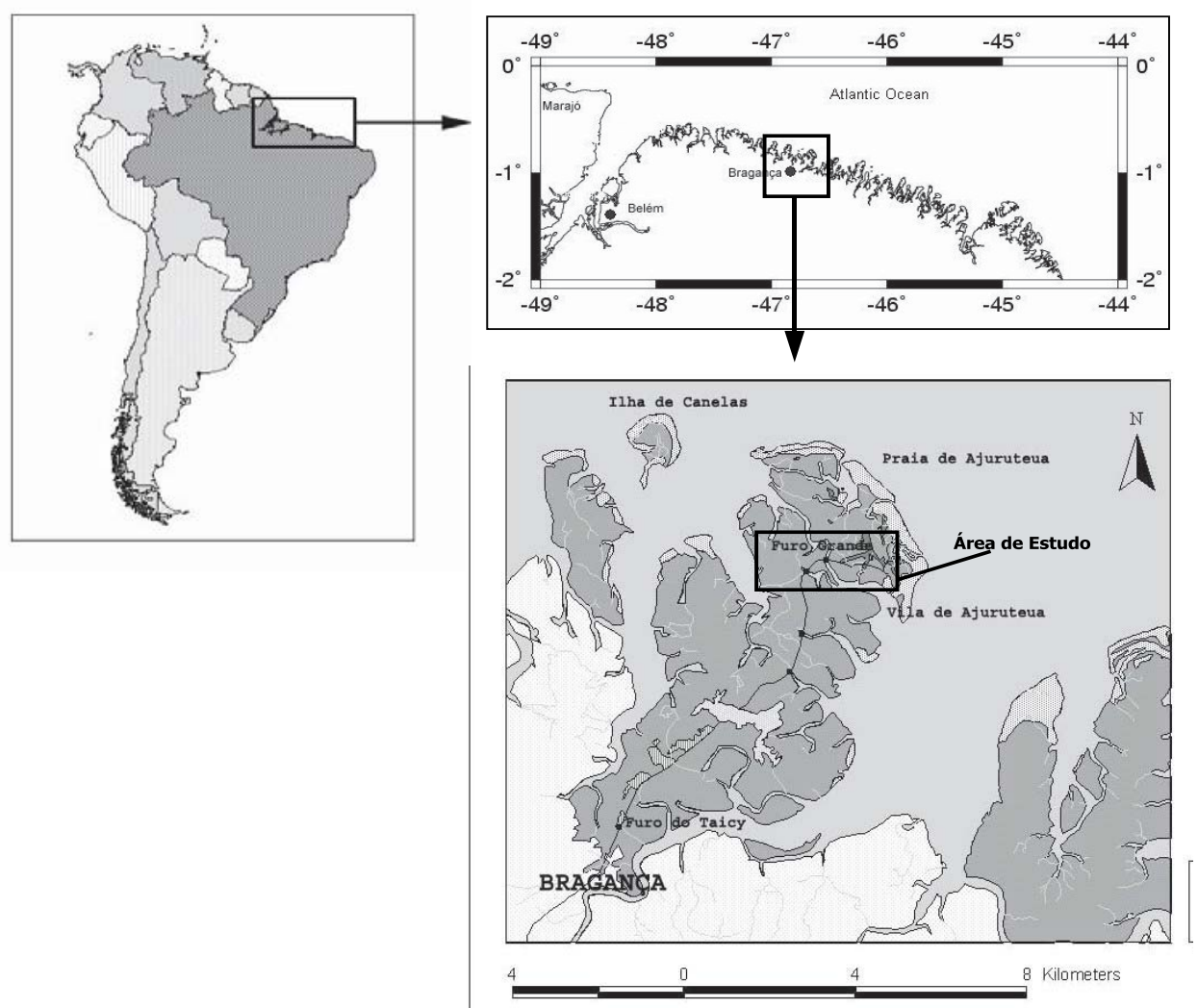

Figura 1 - Mapa mostrando a localização da área de estudo na península de Ajuruteua, Bragança, Pará. Fonte: Modificado de Krause et al., 2001.

Figure 1 - Map showing the location of the study area in the Ajuruteua peninsula, Bragança, Pará. Font: Modified from Krause et al., 2001. 
Tabela 1 - Média da produção anual dos componentes da serapilheira nos três sítios de trabalho (Sítio $1=$ A. germinans e $R$. mangle, Sítio $2=A$. germinans e sítio $3=R$. mangle . DP $=$ desvio-padrão. Nível de significância $(\alpha)=0,05$

Table 1 - Mean annual component litter fall at the three study sites. Site $1=A$. germinans and R. mangle; site $2=A$. germinans and site; $3=R$. mangle; $e S D=$ Standard Deviation, significance level $(\alpha)=0.05$

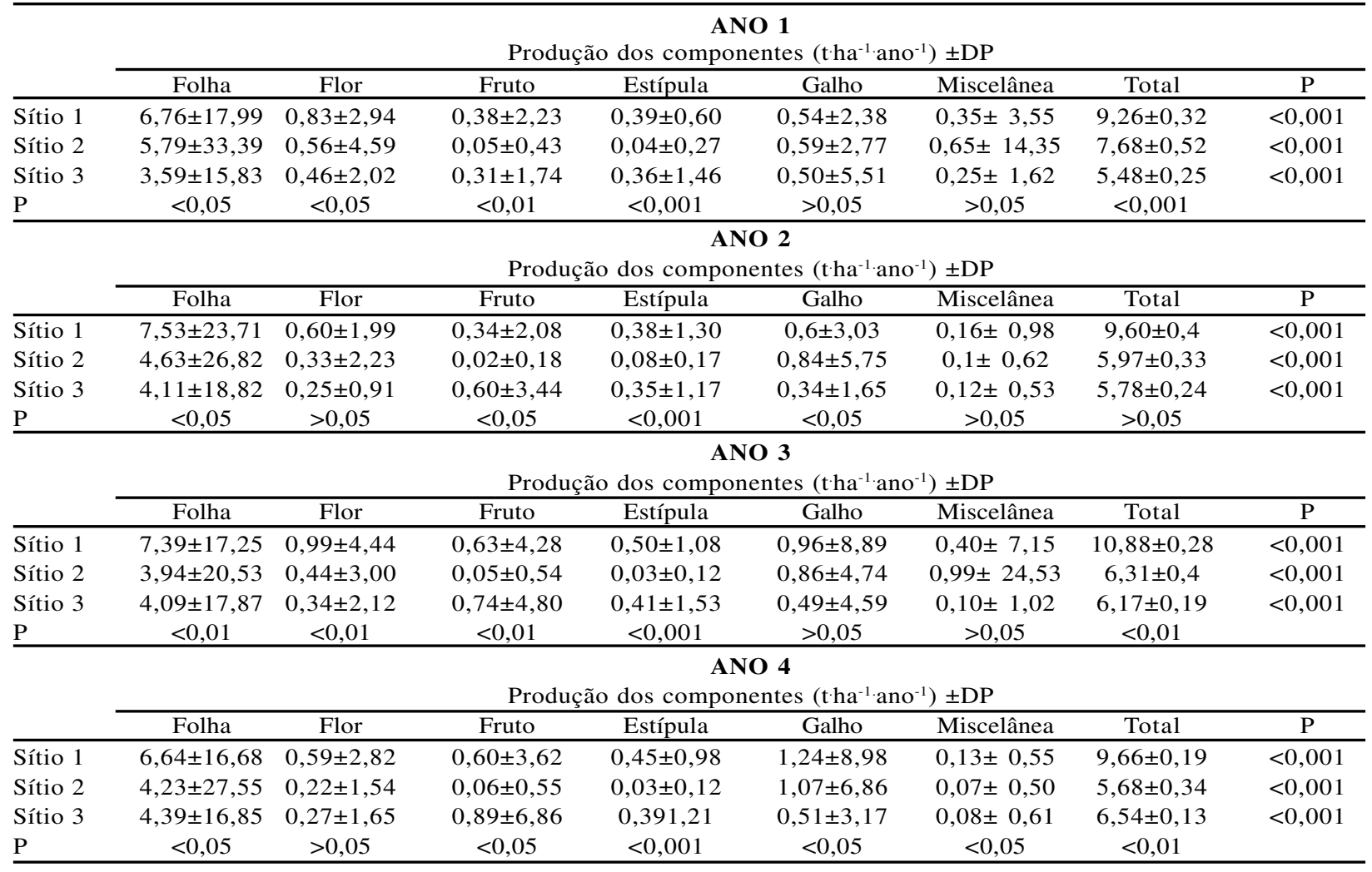

As taxas médias de produção dos componentes da serapilheira para cada sítio de trabalho estão descritas na Tabela 1. Considerando todos os componentes, folha apresentou as maiores médias de produção em todos os sítios, ao passo que estípula exibiu a menor média no sítio 2 em todos os anos e miscelânea, as menores taxas de produção nos sítios 1 e 3 .

A Tabela 1 também apresentou a análise dos valores de produção de cada componente entre os três sítios (análise por coluna), cujos resultados foram significativos, exceto para flor nos anos 2 e 4 (Kruskal-Wallis, $\mathrm{H}=5,79$; $\mathrm{gl}=2 ; \mathrm{p}>0,05-$ ano $2 ; \mathrm{H}=4,40 ; \mathrm{gl}=2 ; \mathrm{e} \mathrm{p}>0,05$ - ano 4), galho nos anos 1 e 3 (Kruskal-Wallis, $\mathrm{H}=4,94$; $\mathrm{gl}=2$; $\mathrm{p}>0,05$ - ano $1 ; \mathrm{e} \mathrm{H}=5,70 ; \mathrm{gl}=2 ; \mathrm{p}>0,05-$ ano 3$)$, miscelânea nos anos 1, 2 e 3 (Kruskal-Wallis, $\mathrm{H}=2,21 ; \mathrm{gl}=2 ; \mathrm{p}>0,05$ - ano $1 ; \mathrm{H}=3,12 ; \mathrm{gl}=2 ; \mathrm{p}>0,05$ - ano 2 e $\mathrm{H}=4,72 ; \mathrm{gl}=2$; $\mathrm{p}>0,05$ - ano 3 ) e a coluna do Total no ano 2 (KruskalWallis, $\mathrm{H}=5,36$; e gl=2; $>>0,05)$. A mesma tabela também apresenta os valores de produção dos componentes por sítio (análise por linha), em que todos os sítios exibiram diferenças significativas (Kruskal-Wallis - sítio $1, \mathrm{H}=59,82 ; \mathrm{gl}=5 ; \mathrm{p}<0,001$ - ano $1 ; \mathrm{H}=63,28 ; \mathrm{gl}=5 ; \mathrm{p}<0,001$ - ano $2 ; \mathrm{H}=59,81 ; \mathrm{gl}=5 ; \mathrm{p}<0,001$ ano $3 \mathrm{e} \mathrm{H}=63,36 ; \mathrm{gl}=5$; $\mathrm{p}<0,001-$ ano 4; Sítio 2, $\mathrm{H}=66,29 ; \mathrm{gl}=5 ; \mathrm{p}<0,001-$ ano $1 ; \mathrm{H}=73,94 ; \mathrm{gl}=5 ; \mathrm{p}<0,001-$ ano $2 ; \mathrm{H}=63,03 ; \mathrm{gl}=5 ; \mathrm{p}<0,001$ - ano 3 e $\mathrm{H}=71,98 ; \mathrm{gl}=5 ; \mathrm{p}<0,001-$ ano 4 e sítio $3, \mathrm{H}=32,07$; $\mathrm{gl}=5 ; \mathrm{p}<0,001-$ ano $1 ; \mathrm{H}=41,75 ; \mathrm{gl}=5 ; \mathrm{p}<0,001-$ ano $2 ; \mathrm{H}=36,62 ; \mathrm{gl}=5 ; \mathrm{p}<0,001-$ ano 3 e $\mathrm{H}=41,94 ; \mathrm{e} \mathrm{gl}=5$; $\mathrm{p}<0,001-$ ano 4$)$.

Quanto à variação sazonal da produção de serapilheira nos três sítios de trabalho, os sítios 1 e 2 apresentaram um padrão semelhante entre si ao longo dos quatro anos de estudo, sendo seus maiores valores de produção no período de maio a novembro, enquanto o sítio 3 mostrou altos valores de produção no período de janeiro a novembro (Figura 2). A análise considerando a variação sazonal de cada sítio mostrou que não houve diferença significativa entre os três sítios (Friedman, p>0,05). 


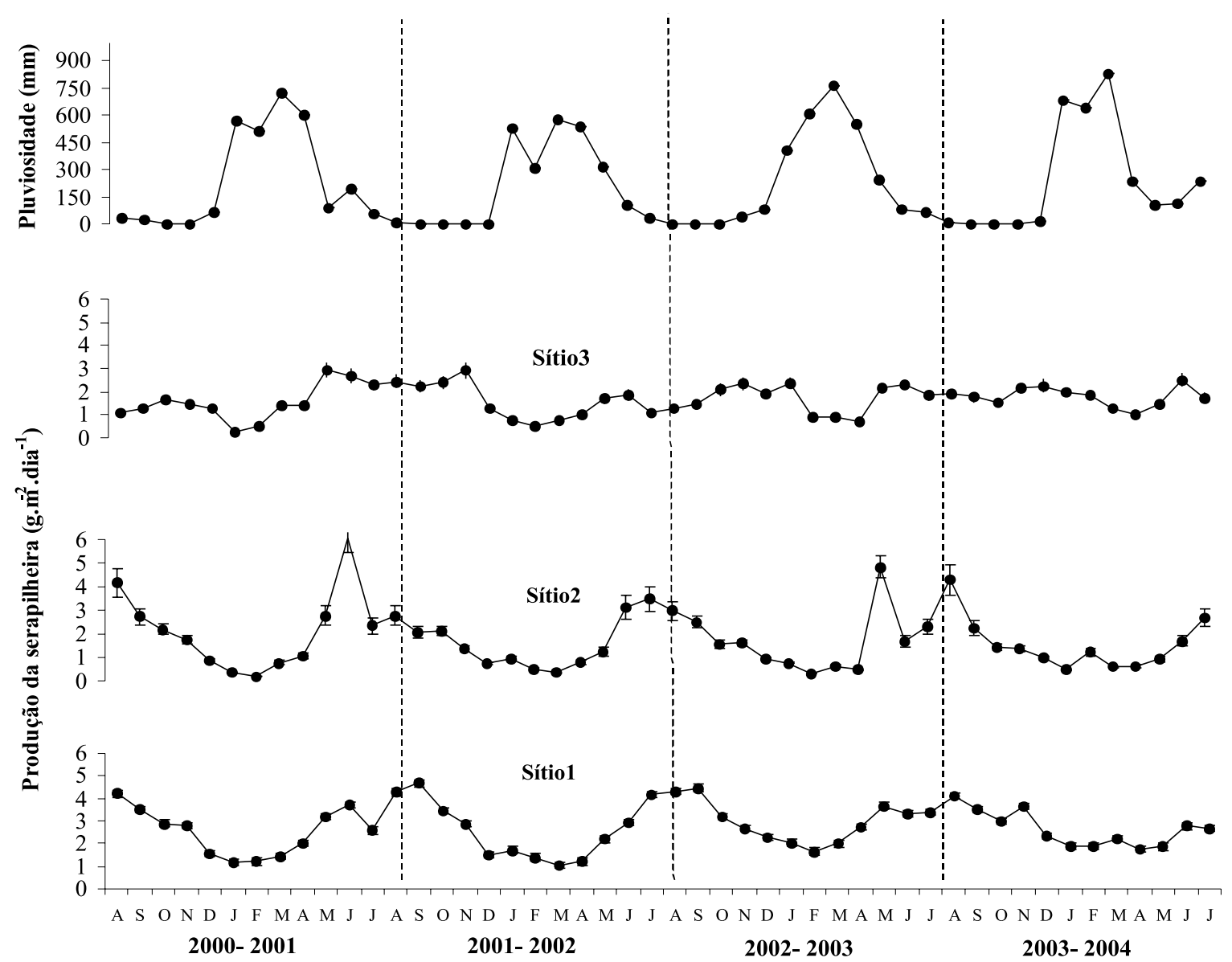

Figura 2 - Variação anual da produção de serapilheira nos três sítios estudados no Furo Grande, Bragança, Pará (sítio 1 = vegetação mista, sítio $2=$ A. germinas e sítio $3=R$. mangle $)$.

Figure 2 - Annual variation of litter fall production for the three studied sites in Furo Grande, Bragança, Pará. site $1=$ mixed vegetation; site $2=$ A.germinas and site $3=R$. mangle .

A Figura 3 apresenta a variação sazonal dos componentes da serapilheira. Os componentes folha e flor exibiram picos de produção de junho a novembro; o componente fruto mostrou um padrão que vai de janeiro a maio. Galho e estípula apresentaram um padrão desordenado, cujo pico de produção ocorre em meses diferentes a cada ano. Por último, o componente miscelânea teve picos de produção elevados nos anos 1 e 3, correspondendo aos meses de junho e maio, respectivamente. Considerando o fator sazonalidade, a análise comparativa entre os componentes indicou que não houve diferença significativa na maioria desses componentes, excetuando flor (Friedman, $\mathrm{Fr}=12,32$; $\mathrm{gl}=3 ; \mathrm{p}<0,01$ ) e miscelânea (Friedman, $\mathrm{Fr}=16,52 ; \mathrm{gl}=3$; $\mathrm{p}<0,001)$.
O componente folha obteve maiores taxas de produção, oscilando de $62 \%$ a $78 \%$, enquanto estípula, como esperado, apresentou os menores valores de contribuição (de 0,47\% a 6,5\%). Assim, o componente folha foi analisado separadamente.

Os resultados apontaram que em cada sítio a espécie arbórea dominante apresentou a maior taxa de produção de folhas (Figura 4). No sítio 1 (misto), por exemplo, a diferença na produção desse componente entre as espécies dominantes não foi significativa $(\mathrm{p}>0,05)$ ao longo do período de estudo. No entanto, no sítio dominado por A. germinans (sítio 2) a quantidade de folhas produzidas por essa espécie exibiu diferença muito significativa com relação às outras espécies (ANOVA fatorial, $\mathrm{F}=51,61 ; \mathrm{gl}=1,88 ; \mathrm{p}<0,001$ ). Da mesma 
forma, o sítio 3 (dominado por R.mangle) também mostrou predominância na produção de folhas dessa espécie, como era esperado $(\mathrm{F}=76,30 ; \mathrm{gl}=1,88 ; \mathrm{p}<0,001)$. Esta análise também demonstrou que, para o componente folha, não houve diferença significativa, considerandose os anos ou os meses como variáveis.

A análise de regressão linear foi utilizada para correlacionar as taxas de produção de serapilheira com as de precipitação. Como resultado, os componentes galho e miscelânea apresentaram correlação baixa e nãosignificativas $\left(\mathrm{F}=0,87 ; \mathrm{gl}=47 ; \mathrm{R}^{2}=0,1 ; \mathrm{p}>0,05\right.$ e $\mathrm{F}=0,09$, $\mathrm{gl}=47 ; \mathrm{R}^{2}=0,002 ; \mathrm{p}>0,05$, respectivamente). Ao contrário, folha, flor e estípula apresentaram correlações mais altas e bastante significativas $\left(\mathrm{F}=46,60, \mathrm{gl}=47 ; \mathrm{R}^{2}=0,50 ; \mathrm{p}<0,001\right.$; $\mathrm{F}=24,36, \mathrm{gl}=47 ; \mathrm{R}^{2}=0,34 ; \mathrm{p}<0,001, \mathrm{eF}=17,23 ; \mathrm{gl}=47 ; \mathrm{eR}^{2}=0,28$; $\mathrm{p}<0,001$, respectivamente). Todas as correlações tiveram tendências negativas, exceto a do componente fruto $(\mathrm{F}=45,81$; $\mathrm{gl}=47 ; \mathrm{R}^{2}=0,49 ; \mathrm{p}<0,001$ ) (Figura 5).

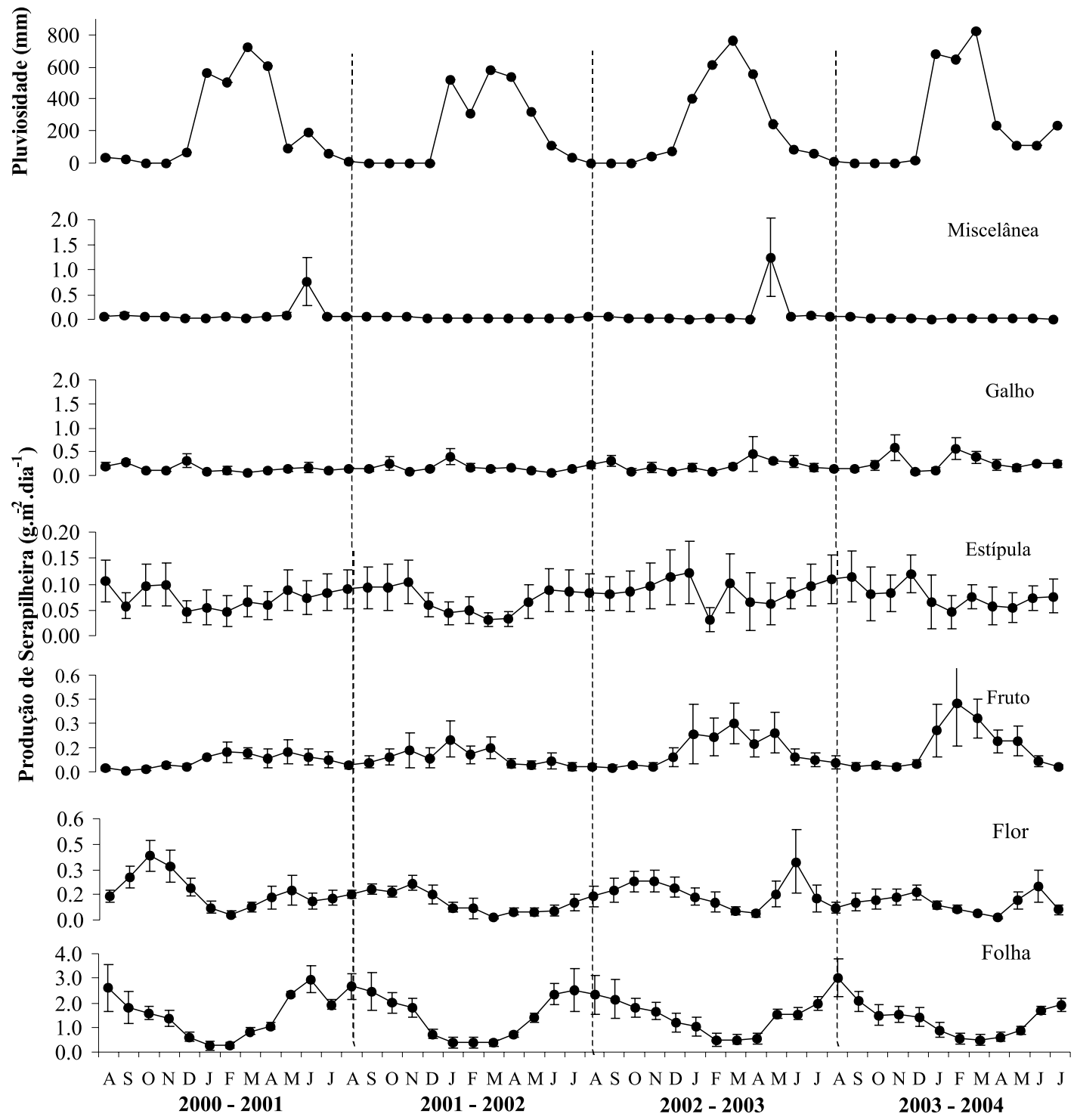

Figura 3 - Variação anual da produção dos componentes da serapilheira nos três sítios estudados no Furo Grande, Bragança, Pará.

Figure 3 - Annual variation in component litter fall production for the three studied sites in Furo Grande, Bragança, Pará.

R. Árvore, Viçosa-MG, v.31, n.5, p.949-958, 2007 


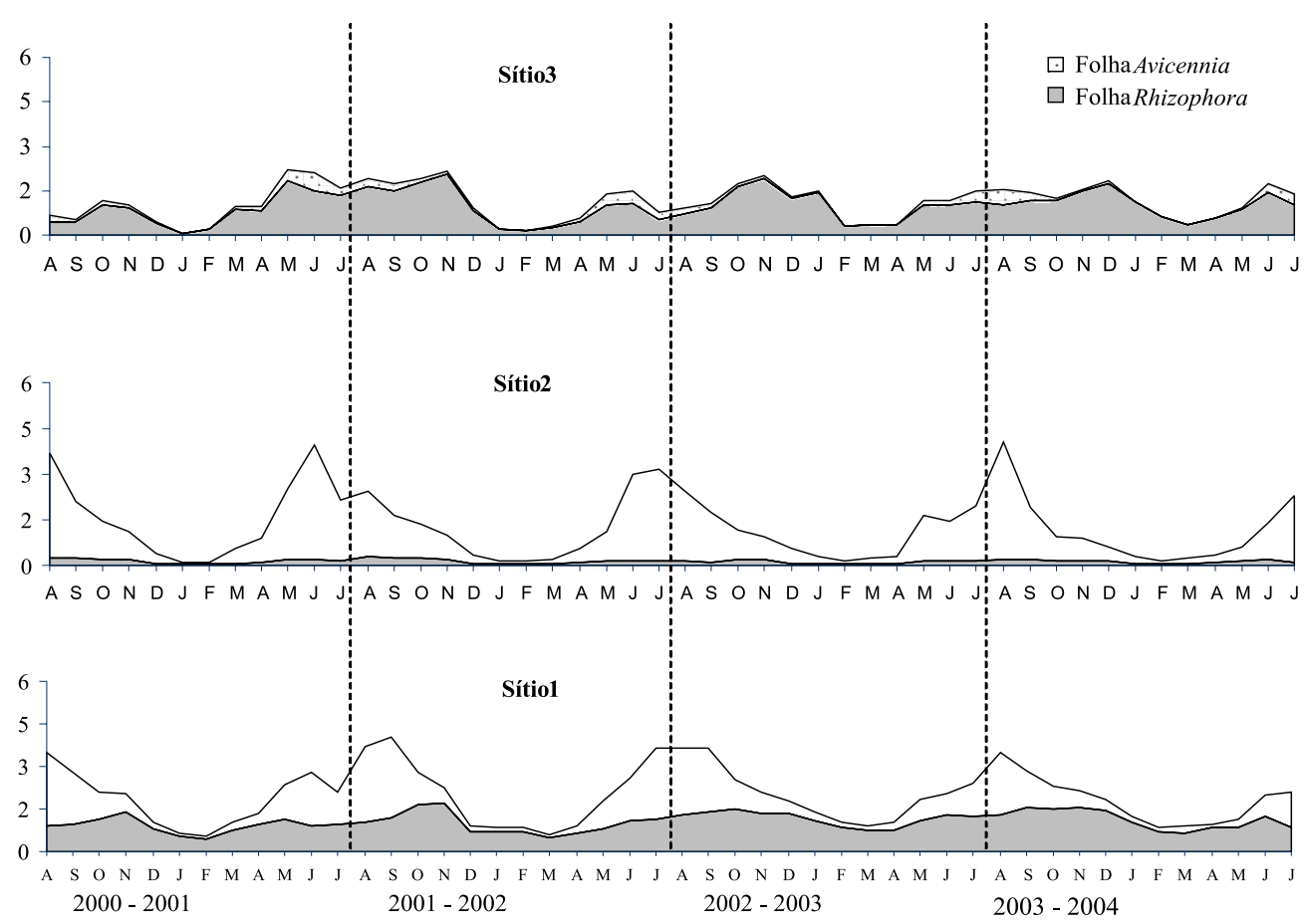

Figura 4 - Variação mensal da produção de folha, no período de agosto/2000 a julho/2004, nos três sítios estudados no Furo Grande, Bragança, Pará.

Figure 4-Monthly variation in leaf production, from August/2000 to July/2004, at the three studied sites in Furo Grande, Bragança, Pará.

\section{DISCUSSÃO}

Neste estudo, a produção de serapilheira apresentou valores totais diferenciados nos três sítios estudados. Tal fato ocorre nas mais diversas áreas de estudo, onde os valores médios de produção de serapilheira, mesmo mostrando condições ambientais similares, são diferentes, como é o caso do estudo realizado na Ilha de Maracá, $\mathrm{AP}$, onde bosques quase monoespecíficos de Rhizophora spp. ou de Avicennia germinans apresentaram variações de até 5 t.ha $^{-1}$.ano ${ }^{-1}$ (FERNANDES, 2003). O mesmo caso foi observado na península de Ajuruteua, onde a produção de serapilheira registrada nos bosques de mangue do Furo do Meio e Acarajó (MEHLIG, 2001), comparada com o valor mostrado neste estudo, atinge, em média, a mesma diferença dos bosques da Ilha de Maracá. No entanto, tais discrepâncias podem ser consequiências de fatores bióticos, a exemplo da estrutura diferenciada das árvores do bosque anão (A. germinans), localizado na península de Ajuruteua, onde a produção alcança apenas $25 \%$ da registrada no presente estudo (REISE, 2003).
Considerando a produção total de serapilheira para cada espécie, observou-se que $A$. germinans foi a mais produtiva. Isso também pode indicar uma provável conseqüência da estrutura dos bosques locais, cuja altura é superior àqueles dominados $R$. mangle (SEIXAS et al., no prelo). Esta última espécie de mangue, praticamente ausente no sítio 3 , foi a que produziu flores e frutos ao longo de todos os anos monitorados, o que também foi um resultado observado por Mehlig (2001) no Furo do Meio e no Acarajó e por Fernandes (2003) na Ilha de Maracá. Contudo, neste trabalho a produção desses componentes (flores frutos) foi contínua apenas nos sítios 1 e 3 , sendo esse fato um reflexo da ausência dessa espécie no sítio 2.

A produção total da serapilheira dos bosques do Furo Grande foi diretamente influenciada pela alta taxa da queda do componente folha, que chegou a alcançar até $78 \%$ do total produzido. Esse componente é considerado um dos mais importantes, por ser uma das principais fontes de matéria orgânica do sistema (SASEKUMAR e LOI, 1983) e de rápida entrada no 
processo de ciclagem de nutrientes (POOL et al., 1975), além de a sua produção ser ininterrupta durante todo o ciclo anual. O pico de produção de folhas foi registrado na estação seca, coincidindo com os resultados obtidos para a produção total de serapilheira de toda a área de estudo (Furo Grande), bem como de outros trabalhos já realizados em manguezais (FERNANDES, 2003; MEHLIG, 2006) e florestas de terra firme (MOREIRA e SILVA, 2004; VITAL et al., 2004).

Os resultados indicaram que a produção de flores e folhas, acumuladas na serapilheira, apresentou padrão similar ao longo dos quatro anos de estudo, registrandose um sincronismo com a estação seca. Esse fato parece evidenciar que a maior abscisão de folhas (queda da serapilheira) pode gerar menor gasto de energia da planta, reduzindo suas taxas fotossintéticas e, conseqüentemente, promovendo maior investimento em reprodução, através da maior produção de flores. Esta sincronia está de acordo com a tendência geral das florestas tropicais, onde a maioria das árvores floresce durante um período de caducifolia (JANZEN, 1967). Ao contrário, o padrão identificado para a produção de frutos, nos manguezais do Furo Grande, apresenta sincronia com o período chuvoso, propiciando a dispersão dos propágulos e sementes das espécies de mangue por hidrocoria. Por fim, tanto os fatores exógenos quanto as adaptações endógenas dessas espécies arbóreas do manguezal parecem interagir, no intuito de garantir a alta produtividade desse sistema e a reprodução desses indivíduos, promovendo o possível estabelecimento em áreas adjacentes, ao longo da península de Ajuruteua.
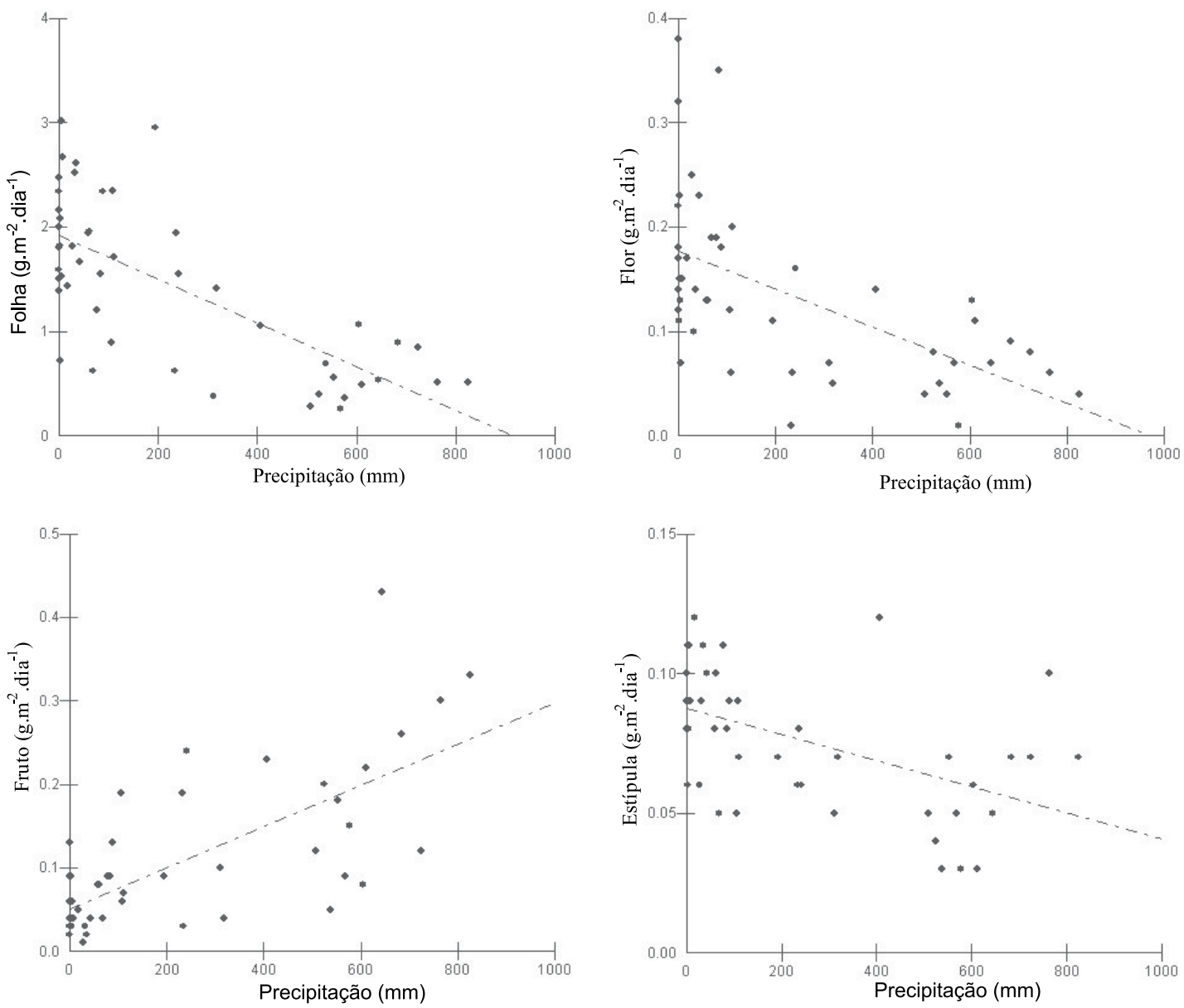

Figura 5 - Relações entre a produção anual de serapilheira por componente e as taxas de precipitação. Figure 5 - Relationships between annual litter fall production per component and the precipitation rates.

R. Árvore, Viçosa-MG, v.31, n.5, p.949-958, 2007 


\section{AGRADECIMENTOS}

Ao Projeto MADAM (Mangrove Dynamics and Management) entre Brasil (CNPq) e Alemanha (ZMT), pelo apoio logístico durante a realização do trabalho de campo e pelo financiamento, através de bolsas do CNPq (ITI-Processo n ${ }^{\circ}$ 190104/2004-0 e DTI -Processo n $390035 / 04-1)$.

\section{REFERÊNCIAS}

AYRES, M. et al. BiosEstat 3.0:

Aplicações estatísticas nas áreas das ciências biológicas e médicas. Belém: Sociedade Civil Mamirauá, 2003. 290p.

AMARASINGHE, M. D.; BALASUBRAMANIAM, $S$. Net primary productivity of two mangrove forest stands on the northwestern coast of Sri Lanka. Hydrobiologia, v.247, p.37-47, 1992.

ARATO, H. D.; MARTINS, S. V.; FERRARI, S. H. S. Produção e decomposição de serapilheira em um sistema agroflorestal implantado para recuperação de área degradada em Viçosa-MG. Revista Árvore, v.27, n.5, p.715-721, 2003.

CHALE, F. M. M. Litter production in an Avicennia germinans (L.) Stearn. forest in Guyana, South America. Hydrobiologia, v.330, n.1, p.47-53, 1996.

DAY Jr., J. W. et al. The productivity and composition of mangrove forests, Laguna de Términos, Mexico. Aquatic Botany, v.27, p.267-284, 1987.

FERNANDES, M. E. B. Produção primária: Serapilheira. In: FERNANDES, M. E. B (Ed.). Os manguezais da costa norte brasileira. São Luís: Fundação Rio Bacanga, 2003. p.61-78.

JANZEN, D. H. Synchronization of sexual reproduction of trees within the dry season in Central America. Evolution, v.21, p.620-637, 1967.

$\mathrm{KOCH}$, V. Epibenthic production and energy flow in the Caeté mangrove estuary, North Brazil. 1999. 97f. Tese (Doutorado em Ecologia) - Zentrum für Marine Tropenökologie, Bremen, 1999.
KRAUSE, G. et al. Spatial patterns of mangroves ecosystems: the bragantinian mangrove of Northern Brazil (Bragança, Pará). Ecotropica, v.7,p.93-107, 2001.

\section{MARTORANO, L. G. et al. Estudos climáticos do Estado do Pará, classificação climática (Köppen) e deficiência hídrica (Thornthwhite, Mather). Belém: SUDAM/Embrapa/SNLCS, 1993. 53p.}

MEHLIG, U. Aspects of tree primary production in an equatorial mangrove forest in Brazil. 2001. 137f. Tese (Doutorado em Ecologia) - Zentrum für Marine Tropenökologie, Bremen, 2001.

MEHLIG, U. Phenology of the red mangrove, Rhizophora mangle L., in the Caeté Estuary, Pará, equatorial Brazil. Aquatic Botany, v.84, n.2, p.158-164, 2006.

MOREIRA, P. R.; SILVA, A. O. Produção de serapilheira em áreas reflorestadas. Revista Árvore, v.28, n.1, p.49-59, 2004.

POOL, D. J.; LUGO, A. E.; SNEDAKER, S. C. Litter production in mangrove forests of southern Florida and Puerto Rico. In: WALSH, G. E.; SNEDAKER, S. C.; TEAS, H. J. (Eds.). INTERNATIONAL.

SYMPOSIUM .BIOLOGY AND
MANAGEMENT OF
MANGROVES, 1975, Florida.

Proceendings...Florida: IFAS, 1975. p.213-299.

REISE, A. Estimates of biomass and productivity in Fringe mangroves of North-Brazil. 2003. 196f. Tese (Doutorado em Ecologia) - Zentrum für Marine Tropenökologie, Bremen, 2003.

SASEKUMAR, A.; LOI, J. J. Litter production in three mangrove forest zones in the Malay peninsula. Aquatic Botany, v. 17, p.283-290, 1983.

R. Árvore, Viçosa-MG, v.31, n.5, p.949-958, 2007 
SAENGER, P.; SNEDAKER, S. C. Pantropical trends in mangrove above-ground biomass and annual litterfall. Oecologia, v.96, p.293-299, 1993.

SEIXAS, J. A. S.; FERNANDES, M. E. B.; SILVA, E. S. Análise estrutural da vegetação arbórea dos mangues no Furo Grande, Bragança - Pará. Boletim do Museu Paraense Emílio Goeldi Ciências Naturais, v.1, n.3, p.61-69, 2006.
SILVA, M. F. F. Produção anual de serapilheira e seu conteúdo mineralógico em mata tropical de terra firme, Tucuruí-PA. Boletim do Museu Paraense Emílio Goeldi Ser. Bot., v.17,n.1/2, p.111-158, 1984.

VITAL, A. R. T. et al. Produção de serapilheira e ciclagem de nutrientes de uma floresta estacional semidecidual em zona ripária. Revista Árvore, v.28, n.6, p.793-800, 2004. 